\title{
Analysis of Negativity and Relative Entropy of Entanglement Measures for Two Qutrit Quantum Communication Systems
}

\author{
Volkan Erol \\ Computer Engineering Department, Okan University, Netaş Telekomünikasyon, İstanbul, Turkey; \\ volkan.erol@gmail.com; Tel.: +90-533-3621947
}

\begin{abstract}
Quantum Computers are provisioned as very high performance computers using quantum mechanical aspects for information processing. Quantum Information Theory and Quantum Computing topics are popular topics in academia aiming the construction of theoretical background of Quantum Computers. The information processing units for Quantum Computers are defined as qubits but for some problems three level (trinary) systems may be applied as well. We call these three level systems as qutrits. The scope of this work is the analysis of well-defined entanglement measures Negativity and Relative Entropy of Entanglement (REE) for two qutrit (3- level/trinary) quantum systems. In this manner, for randomly generated 1000 two qutrit and 1000 two qubit states the mentioned measures are calculated and these values are compared. These comparisons are analyzed and for quantum state ordering problem, some interesting results are reported.
\end{abstract}

Keywords: entanglement; qubit; qutrit; concurrence; negativity; relative entropy of entanglement

\section{Introduction}

Thanks to quantum entanglement, quantum mechanical systems can make a lot of information processing task much more quickly than the classical mechanical systems. For example Shors factorization algorithm, Grovers search algorithm, Quantum Fourier Transformation etc. [1-9]. On the other hand, lot of work such as the quantum teleportation, super dense coding and quantum key distrubution $(\mathrm{QKD})$ are not possible with classical mechanical systems, they are only possible with quantum mechanical counterparts. Therefore, quantum technologies are in an exploration era for areas like computer science, communication and encryption.

Quantum entanglement can be observed in bipartite and multi-partite forms. Various criteria has been developed to measure the entanglement for bilateral systems. For example, Negativity, Logarithmic Negativity and Concurrence depend on the eigenvalues of the partial transposition of the system's density matrix. Relative Entropy of Entanglement (REE) is based on the distance of the nearest system which is not entangled to the mentioned system.

Quantum Fisher Information (QFI) is a measure of a quantum system parameter's sensitivity for changes. If we investigate QFI of a system's state per particle, we obtain shot-noise limit information for separable states [10]. QFI topic is mainly studied in many recent works both as theoretically and experimentally [11-16, 31-36].

On the other hand, a general and accepted multipartite entanglement measure is not defined so far. Furthermore, since multipartite entanglement creation and processing may be used in computation problems, it is one of the hot topics in quantum information domain [17-36]. QFI may be considered a first general entanglement measure for multipartite systems and some meaningful research activities continue in this aspect [23-36]. 
Our added value in this work could be explained as follows: we compare widely studied negativity and relative entropy of entanglement measure for 1000 random states for general two level (qubit) and three level (qutrit) systems. In this manner, we analyse these values respectively and obtain some interesting results and report them.

\section{Materials and Methods}

Negativity is a quantitative version of Peres-Horodecki criterion [19]. It is defined for two particle two level general quantum systems as follows [2,20,21]:

$$
N(\varrho)=\max \{0,-2 \mu \min \},
$$

Here $\mu_{\min }$ value is the minimum eigenvalue of Q's partial transpose. Negativity, which is defined by the equation above is a value between 0 and 1 like Concurrence. Similarly like for concurrence, 1 means maximal entanglement.

Vidal and Werner shown that Negativity is a monotone function for entanglement [21].

For calculating concurrence values of two qutrit systems there is no closed formula. Because of this situation, there are some derivatives of concurrence for these systems. Therefore, for measuring two qutrit systems' entanglement in general we use Negativity. Negativity is calculated in a similar way for two qutrit systems [30]:

$$
\mathrm{N}\left(\mathrm{Q}^{\mathrm{AB}}\right)=\max \left\{0,-2 \mu_{\min }\right\},
$$

Here $\mu$ min value is the minimum eigenvalue of $\mathrm{Q}^{\mathrm{AB}^{\prime}}$ s partial transpose due to system $\mathrm{A}$.

Relative Entropy of Entanglement (REE) is a measure based on the distance of the state to the closest separable state. Mathematically it can be defined as follows: the minimum of the quantum relative entropy $S(\varrho|| \sigma)=\operatorname{Tr}(\varrho \log \varrho-\varrho \log \sigma)$ taken over the set $D$ of all separable states $\sigma$, namely for each $\mathrm{Q}$ in $\mathrm{D}$

$$
\mathrm{E}(\varrho)=\min (S(\varrho|| \sigma))=S\left(\varrho|| \sigma^{\prime}\right)
$$

where $\sigma^{\prime}$ denotes the closest state to @.

For this measure there is no closed formula found for two-level or morelevel systems. For some specific and multi-level systems there are some formula suggestions. For two-level systems there are some estimations based on semidefinite programming [37]. We used the software provided in [37] to calculate REE values.

\section{Results}

In this section we show our results for random 1000 two-qubit (two-level) and 1000 qutrit (threelevel) systems' Negativity and REE measures. Results are given in Figures 1 and 2. 


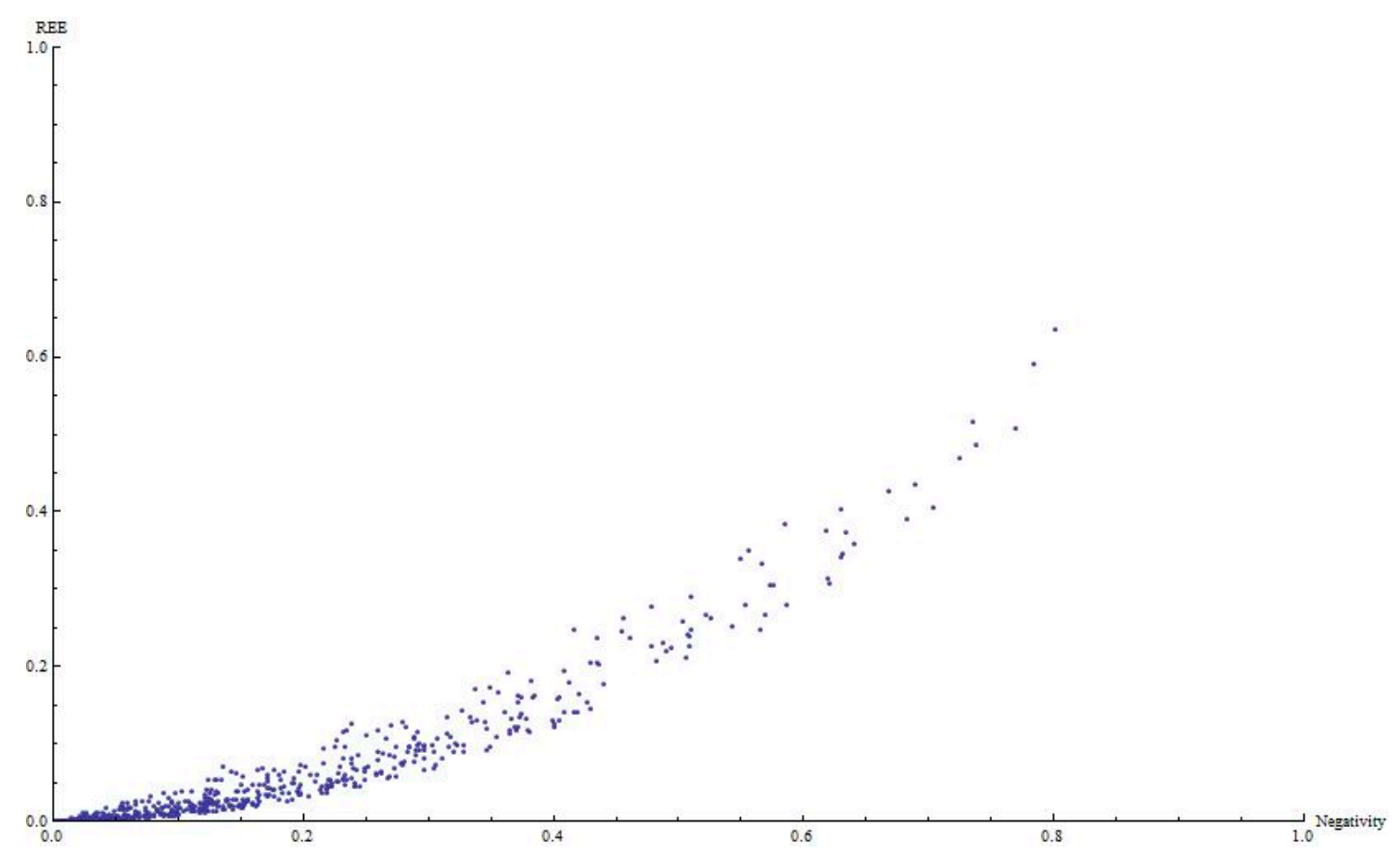

Figure 1. Results for two qubit systems

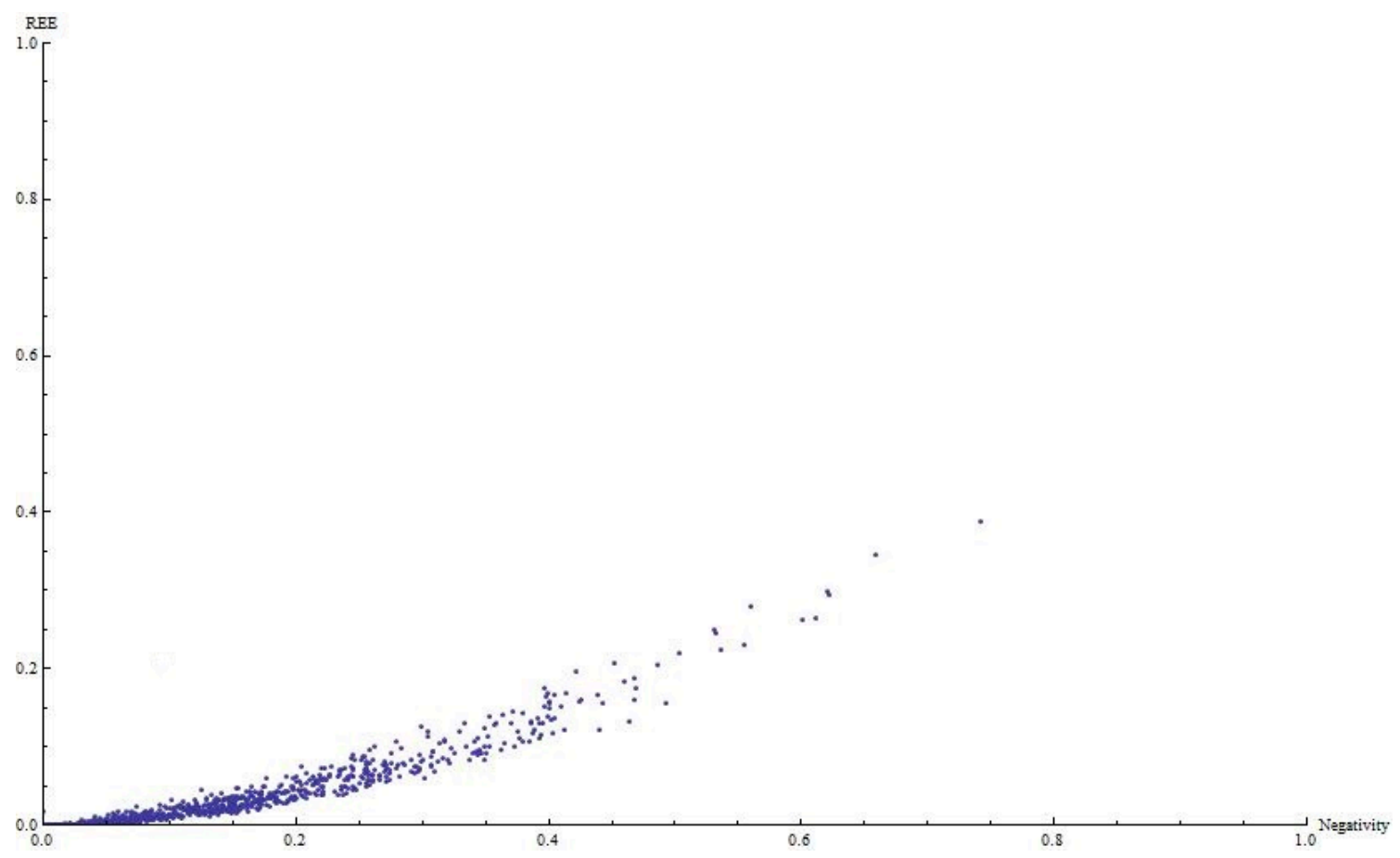

Figure 2. Results for two qutrit systems

Table 1. Comparison of Negativity and REE

\begin{tabular}{rc}
\hline Class & Neg vs REE \\
\hline 1 & $\mathrm{~N}\left(\mathrm{~S}_{1}\right)>\operatorname{REE}\left(\mathrm{S}_{1}\right)$ \\
\hline
\end{tabular}

\section{Discussion}

As a result of the comparison is made, two qubit and two qutrit system states which have reel density matrices, generally Negativity values are larger than REE values have been estimated. 
In each case, almost exactly same graphic has been occured. This is quite an interesting result for sorting of system states. In this case, only one class for sorting problem has been determined. This result is not only [3] and [4] scope, also it is quite important for Quantum Information Theory and Ouantum Communication.

If bipartite larger entangled systems will be examined in future studies, bound entangled system states can be estimated are thought of will be much more different comparison graphics.

There is a closed formula for Negativity measure in two or more level systems, but for REE measure there is no closed formula found, there are estimation methods for two level and three level systems as it is not there is no estimation method for larger systems. This is an open research and it can be studied by researchers.

\section{References}

1. M.A. Nielsen and I.L. Chuang, Quantum Computation and Quantum Information, Cambridge, UK: Cambridge Univ. Press, 2000.

2. J. Eisert and M. B. Plenio, A comparison of entanglement measures, J. Mod. Opt., vol.84, pp. 145-154, 1999.

3. A. Miranowicz and A. Grudka, Ordering two-qubit states with concurrence and negativity, Phys. Rev. A, vol. 70, no. 032326, 2004.

4. A. Miranowicz and A. Grudka, A comparative study of relative entropy of entanglement, concurrence and negativity, J. Opt. B: Quantum Semiclass. Opt. vol. 6, pp. 542548, 2004.

5. M. Koashi and A. Winter, Monogamy of quantum entanglement and other correlations, Phys. Rev. A, vol. 69, no. 022309, 2004.

6. B. Horst, K. Bartkiewics and A. Miranowicz, Two-qubit mixed states more entangled than pure states: Comparison of the relative entropy of entanglement for a given nonlocality, Phys. Rev. A, vol. 87, no. 042108, 2013.

7. M. B. Plenio, Logarithmic Negativity: A Full Entanglement Monotone That is not Convex, Phys. Rev. Lett., vol. 95, no. 090503, 2005.

8. K. Zyczkowski et al., Volume of the set of separable states, Phys. Rev. A, vol. 58, no.883, 1998.

9. A. Zeilinger et al., Three-Particle Entanglements from Two Entangled Pairs, Phys. Rev. Lett., vol. 78, no. 3031, 1997.

10. L. Pezze, and A. Smerzi, Entanglement, Nonlinear Dynamics, and the Heisenberg Limit, Phys. Rev. Lett., vol. 102, no. 100401, 2009.

11. Z. Ji et al., Parameter Estimation of Quantum Channels IEEE Trans. Inf. Theory., vol. 54, no. 5172, 2008.

12. B.M. Escher, M. Filho, and L. Davidovich, General framework for estimating the ultimate precision limit in noisy quantum-enhanced metrology, Nat. Phys., vol. 7, no. 406, 2011.

13. N. Spagnalo et al., Quantum interferometry with threedimensional geometry, Sci. Rep., vol. 2, no. 862, 2010. 
14. M. Kacprowicz et al., Experimental quantum-enhanced estimation of a lossy phase shift, Nat. Photon., vol 4, no. 357, 2010.

15. F. Ozaydin et al., Quantum Fisher Information of N Particles in the Superposition of W and GHZ States, Int. J. Theor. Phys, vol. 52, no. 2977, 2013.

16. F. Ozaydin et al., Behavior of Quantum Fisher Information of Bell Pairs under Decoherence Channels, Acta Physica Polonica A, vol. 125 (2), no. 597, 2014

17. T. Tashima et al., Elementary optical gate for expanding an entanglement web, Phys. Rev. A, vol. 77, no. 030302, 2008.

18. T. Tashima et al., Local expansion of photonic W state using a polarization-dependent beamsplitter, New J. Phys. A, vol. 11, no. 023024, 2009.

19. T. Tashima et al., Local Transformation of Two EinsteinPodolsky-Rosen Photon Pairs into a Three-Photon W State, Phys. Rev. Lett. , vol. 102, no. 130502, 2009.

20. S. K. Ozdemir et al., An optical fusion gate for W-states, New J. Phys., vol. 13, no. 103003, 2011.

21. S. Bugu, C. Yesilyurt and F. Ozaydin, Enhancing the W-state quantum-network-fusion process with a single Fredkin gate, Phys. Rev. A, vol. 87, no. 032331, 2013.

22. C. Yesilyurt, S. Bugu and F. Ozaydin, An Optical Gate for Simultaneous Fusion of Four Photonic W or Bell States, Quant. Info. Proc., vol. 12, no. 2965, 2013.

23. P. Hyllus et al., Fisher information and multiparticle entanglement, Phys. Rev. A, vol. 85, no. 022321, 2012.

24. V. Erol, F. Ozaydin and A. A. Altintas, Analysis of Entanglement Measures and LOCC Maximized Quantum Fisher Information of General Two Qubit Systems, Sci. Rep., 4, 5422, (2014).

25. V. Erol, S. Bugu, F.Ozaydin and A. A. Altintas, An analysis of concurrence entanglement measure and quantum fisher information of quantum communication networks of two-qubits, 2014 IEEE 22nd Signal Processing and Communications Applications Conference (SIU 2014), 317-320 (2014).

26. F. Ozaydin, A. A. Altintas, C. Yesilyurt, S. Bugu and V. Erol, Quantum Fisher Information of Bipartitions of W States, Acta Physica Polonica A, 127(4), 1233 (2015).

27. V. Erol, Kuantum Fisher Bilgisi Optimizasyonu Onerisi ve Dolaniklik Olcuttleri ile Iliskisi, Okan University, FBE (2015).

28. V. Erol, F.Ozaydin and A. A. Altintas, Analysis of Negativity and Relative Entropy of Entanglement measures for qubit-qutrit Quantum Communication systems, 2015 IEEE 23nd Signal Processing and Communications Applications Conference (SIU 2015), 116-119 (2015).

29. V. Erol, A comparative study of concurrence and negativity of general three-level quantum systems of two particles, AIP Conf. Proc. 1653 (020037).

30. G. Karpat, Z. Gedik, "Correlation dynamics of qubitqutrit systems in a classical dephasing environment", Phys. Let. A. 375, 41664171 (2011).

31. F. Ozaydin, "Phase damping destroys quantum Fisher information of W states", Phys. Let. A. 378, 3161-3164 (2014). 
32. F. Ozaydin, S. Bugu, C. Yesilyurt, A.A. Altintas, M. Tame, S.K. Ozdemir, Fusing multiple W states simultaneously with a Fredkin gate, Phys. Rev. A 89, 042311 (2014).

33. A.A. Altintas, Quantum Fisher information of an open and noisy system in the steady state, Annals of Physics, DOI: 10.1016/j.aop.2016.01.016

34. F. Ozaydin., Quantum Fisher Information of a 33 Bound Entangled State and its Relation with Geometric Discord, Int. J. Theor. Phys, vol. 54(9), 3304-3310, 2015.

35. F. Ozaydin and A. A. Altintas, Quantum Metrology: Surpassing the shot-noise limit with DzyaloshinskiiMoriya interaction, Sci. Rep., 5, 16360, (2015).

36. X.P. Zang, M. Yang, F. Ozaydin, W. Song and Z.L. Cao Generating multi-atom entangled W states via lightmatter interface based fusion mechanism, Sci. Rep., 5, 16245,(2015).

37. Y. Zinchenko, S. Friedland and G. Gour, Numerical estimation of the relative entropyof entanglement, Phys. Rev. A 82, 052336 (2010).

(C) 2017 by the author. Licensee Preprints, Basel, Switzerland. This article is an open access article distributed under the terms and conditions of the Creative Commons by Attribution (CC-BY) license (http://creativecommons.org/licenses/by/4.0/). 